\title{
Implications of Adler Zeros for Multipion Processes.
}

\author{
H. OSBORN \\ Department of Physics, Queen Mary College - London \\ (Lett. Nuovo Cimento, 2, 717 (1969))
}

The proof of the theorem enunciated in this paper is not complete as it stands. In view of the relation

$$
\boldsymbol{\pi}^{2} \boldsymbol{\pi} \cdot \square \pi=\boldsymbol{\pi} \cdot \square\left(\boldsymbol{\pi} \boldsymbol{\pi}^{2}\right)-\frac{1}{2} \square \boldsymbol{\pi}^{4}
$$

it is possible in any multipion irreducible part for a linear dependence on the (pion mass) ${ }^{2}$ invariants to be eliminated in favour of a dependence on the Mandelstam energy variables for $3 \pi$ 's in an $I=1$ state and $4 \pi$ 's in an $I==0$ state, which are not exotic. With generalizations of the above and the results of the paper any linear expansion of a $2 n \pi$ irredueible part in any invariants can be written in terms of the Mandelstam variables for $2 m \pi$ 's in $I=0$ and $(2 m-1) \pi$ 's in $I=1$ states for any $m n$. To rule out interactions of type $(2 c)$ or $(3 c)$ it is necessary to postulate no dependence on Mandelstam variables for pions in an exotic state or with the quantum numbers, $G=-, J^{p}=0^{-}$, of a single pion in any irreducible part. This is in accord with duality since it can then be assumed that the pion poles in the multipion amplitudes constructed under the conditions of the theorem generate the entire dependence on the Mandelstam variables for states with pion quantum numbers. However the Frye-Susskind conjecture is unaffected since the above result is valid only when summed over all permutations of the external pions, and not for just cyclic and anticyclic permntations as occur in each term of the algebraic duality expansion, so the proof of the conjecture given still holds. I am grateful to J. ElLIs for pointing this out.

There is a slight misprint in the second line following eq. (8). The first $\pi$ should have a tilde and the second should be bold face. 